Louisiana State University

LSU Digital Commons

Faculty Publications

Department of Physics \& Astronomy

8-21-2019

\title{
First measurements of the spectral and angular distribution of transition radiation using a silicon pixel sensor on a Timepix 3 chip
}

\author{
E. J. Schioppa \\ European Organization for Nuclear Research \\ F. Dachs \\ European Organization for Nuclear Research \\ J. Alozy \\ European Organization for Nuclear Research \\ N. Belyaev \\ National Research Nuclear University MEPhI \\ M. Campbell \\ European Organization for Nuclear Research
}

See next page for additional authors

Follow this and additional works at: https://digitalcommons.Isu.edu/physics_astronomy_pubs

\section{Recommended Citation}

Schioppa, E., Dachs, F., Alozy, J., Belyaev, N., Campbell, M., Cherry, M., Doronin, S., Filippov, K., Fusco, P., Gargano, F., Heijne, E., Konovalov, S., Krasnopevtsev, D., Llopart, X., Loparco, F., Mascagna, V., Mazziotta, M., Pernegger, H., Ponomarenko, D., Prest, M., Pyatiizbyantseva, D., Radomskii, R., Rembser, C., Romaniouk, A., Shulga, E., Smirnov, S., Smirnov, Y., Soldani, M., Spinelli, P., Sergeeva, D., Savchenko, A., Schaefer, D., \& Strikhanov, M. (2019). First measurements of the spectral and angular distribution of transition radiation using a silicon pixel sensor on a Timepix3 chip. Nuclear Instruments and Methods in Physics Research, Section A: Accelerators, Spectrometers, Detectors and Associated Equipment, 936, 523-526. https://doi.org/10.1016/j.nima.2018.11.062

This Article is brought to you for free and open access by the Department of Physics \& Astronomy at LSU Digital Commons. It has been accepted for inclusion in Faculty Publications by an authorized administrator of LSU Digital Commons. For more information, please contact ir@lsu.edu. 


\section{Authors}

E. J. Schioppa, F. Dachs, J. Alozy, N. Belyaev, M. Campbell, M. Cherry, S. Doronin, K. Filippov, P. Fusco, F. Gargano, E. H.M. Heijne, S. Konovalov, D. Krasnopevtsev, X. Llopart, F. Loparco, V. Mascagna, M. N. Mazziotta, H. Pernegger, D. Ponomarenko, M. Prest, D. Pyatiizbyantseva, R. Radomskii, C. Rembser, A. Romaniouk, E. Shulga, S. Smirnov, Y. Smirnov, M. Soldani, P. Spinelli, D. Yu Sergeeva, A. A. Savchenko, D. Schaefer, and M. Strikhanov 


\section{First measurements of the spectral and angular distribution of transition radiation using a silicon pixel sensor on a Timepix3 chip}

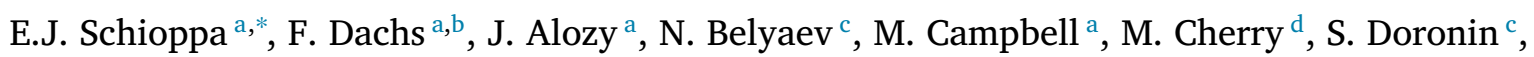
K. Filippov ${ }^{\text {c }}$, P. Fusco e,f, F. Gargano ${ }^{\text {f }}$, E.H.M. Heijne ${ }^{\mathrm{a}}$, S. Konovalov ${ }^{\mathrm{h}}$, D. Krasnopevtsev ${ }^{\mathrm{c}}$, X. Llopart $^{\mathrm{a}}$, F. Loparco e,f, V. Mascagna ${ }^{\mathrm{g}, \mathrm{l}}$, M.N. Mazziotta ${ }^{\mathrm{f}}$, H. Pernegger ${ }^{\mathrm{a}}$, D. Ponomarenko ${ }^{\mathrm{c}}$, M. Prest ${ }^{\text {g,l }}$, D. Pyatiizbyantseva ${ }^{c}$, R. Radomskii ${ }^{c}$, C. Rembser ${ }^{\mathrm{a}}$, A. Romaniouk ${ }^{\mathrm{c}}$, E. Shulga ${ }^{\mathrm{c}}$, S. Smirnov c, Y. Smirnov c , M. Soldani ${ }^{\mathrm{g}, 1}$, P. Spinelli e,f , D. Yu. Sergeeva ${ }^{\mathrm{c}, \mathrm{m}}$, A.A. Savchenko ${ }^{\mathrm{c}, \mathrm{m}}$, D. Schaefer ${ }^{i}$, M. Strikhanov ${ }^{c}$, A.A. Tishchenko ${ }^{c, m}$, P. Teterin ${ }^{c}$, V. Tikhomirov ${ }^{h}$, E. Vallazza ${ }^{j}$, M. van Beuzekom ${ }^{\mathrm{k}}$, B. van der Heijden ${ }^{\mathrm{k}}, \mathrm{K}$. Vorobev ${ }^{\mathrm{c}}$, K. Zhukov ${ }^{\mathrm{h}}$

a CERN, The European Organization for Nuclear Research, Route de Meyrin, 1211 Geneva, Switzerland

b Techincal University of Vienna, Karlsplatz 13, 1040 Vienna, Austria

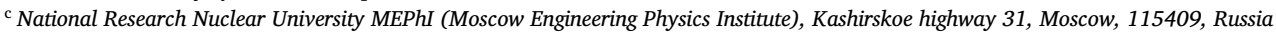

d Department of Physics \& Astronomy, Louisiana State University, Baton Rouge, LA 70803, USA

e Dipartimento di Fisica “M. Merlin" dell'Università e del Politecnico di Bari, Via G. Amendola 173, 70126 Bari, Italy

${ }^{\mathrm{f}}$ Istituto Nazionale di Fisica Nucleare, Sezione di Bari, Via E. Orabona 4, 70126 Bari, Italy

I INFN Milano Bicocca, Piazza della Scienza 3, 20126 Milano, Italy

${ }^{\text {h }}$ P. N. Lebedev Physical Institute of the Russian Academy of Sciences, Leninsky prospect 53, Moscow, 119991, Russia

${ }^{i}$ University of Chicago, 5801 S Ellis Ave, Chicago, IL 60637, USA

j INFN Trieste, Padriciano 99, 34149 Trieste, Italy

${ }^{\mathrm{k}}$ Nikhef, Science Park 105, 1098 XG Amsterdam, The Netherlands

${ }^{1}$ Universitá degli Studi dell'Insubria, Via Valleggio 11, 22100, Como, Italy

m National Research Center "Kurchatov Institute”, Akademika Kurchatova pl. 1, Moscow, Russia

\section{A R T I C L E I N F O}

\section{Keywords:}

Pixel detectors

Transition radiation

Particle identification

\begin{abstract}
A B S T R A C T
X-ray Transition radiation detectors (TRDs) are used for particle identification in both high energy physics and astroparticle physics. Particle identification is often achieved based on a threshold effect of the X-ray transition radiation (TR). In most of the detectors, TR emission starts at $\gamma$ factors above $\sim 500$ and reaches saturation at $\gamma \sim 2-3 \cdot 10^{3}$. However, many experiments require particle identification up to $\gamma \sim 10^{5}$, which is difficult to achieve with current detectors, based only on the measurement of the photon energy together with the particle ionization losses. Additional information on the Lorentz factor can be extracted from the angular distribution of TR photons. TRDs based on pixel detectors give a unique opportunity for precise measurements of spectral and angular distributions of TR at the same time. A $500 \mu \mathrm{m}$ thick silicon sensor bump bonded to a Timepix3 chip was used in a test beam measurement at the CERN SPS. A beam telescope was employed to separate clusters produced by the primary beam particles from the potential TR clusters. Spectral and angular distributions of TR were studied with high precision for the first time using beams of pions, electrons and muons at different momenta. In this paper, the measurement and analysis techniques are described, and first results are presented.
\end{abstract}

Contents

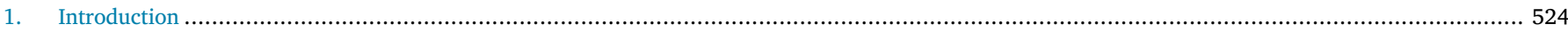

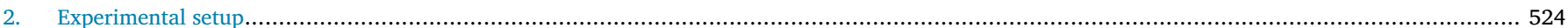

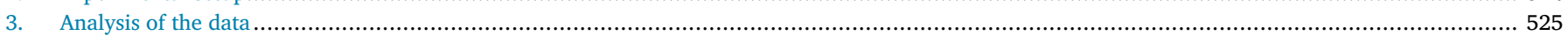

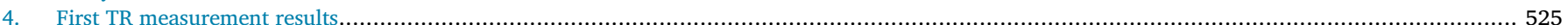

\footnotetext{
* Corresponding author.

E-mail address: enrico.junior.schioppa@cern.ch (E.J. Schioppa).
} 


\section{Introduction}

Transition radiation (TR) is a form of electromagnetic radiation produced by charged particles crossing a boundary between media of different dielectric constants. In the ultra-relativistic case, TR is emitted in the X-ray energy range. The emission angle is a few mrad, so TR can be found in the forward direction close to the particle trajectory. The number of quanta is proportional to the fine structure constant $\left(\sim 10^{-2}\right.$ per interface) and, in practice, radiation produced by multiple surfaces (stack of foils, foam, fibers) is used to increase the yield. The TR intensity for a single boundary crossing increases with $\gamma$, but for multiple boundary crossings, interference leads to saturation above a Lorentz factor of $\gamma_{\text {sat }}=0.6 \omega_{1} \sqrt{\ell_{1} \ell_{2}} / c$, where $\omega_{1}$ is the radiator material plasma frequency, $\ell_{1}$ is the thickness of the layers, and $\ell_{2}$ the spacing between layers. In most of the TRDs used in particle physics, the radiator parameters are chosen to enhance the separation between electrons and pions up to $\gamma$-factors of $\sim 500$ for pions. These detectors normally work as threshold devices, ensuring the best electron/pion separation in the momentum range $1 \mathrm{GeV} / c<p<50 \mathrm{GeV} / c$ (see reviews [1$3]$ and references there). However, many experiments require particle identification up to $\gamma \sim 10^{5}$. By varying the radiator parameters, one may optimize particle separation for a given range of the $\gamma$-factor.

The probability density function of TR produced at a multilayer radiator is a complex function of irradiation angle $\theta$ and photon energy $\omega$. It depends as well on the particle $\gamma$ factor and the radiator parameters. When integrated over the angle, the TR spectrum exhibits several minima and maxima. Most of the radiation is emitted near the last maximum of the spectrum at $\omega_{\max }=\ell_{1} \omega_{1}^{2} / 2 \pi c$, with the effective TR photon emission starting at about $\gamma_{t h r}=\ell_{1} \omega_{1} / c$. The radiation production at each maximum has its own $\gamma$-factor dependence. This feature can be exploited to develop a single detector, with different responses in different Lorentz factor regions [4]. In order to maximize the particle identification capability, future detectors must be capable of extracting the maximum information about the produced TR photons, e.g. the emission angle. The angular distribution of TR photons has a few maxima and extends up to $\theta_{\max }=\left(1 / \gamma^{2}+\omega_{1}^{2} / \omega^{2}\right)^{1 / 2}$ (see [5]). For single foil radiators, the largest fraction of the TR energy is emitted around the most probable angle $\theta=\left(1 / \gamma^{2}+\omega_{2}^{2} / \omega^{2}\right)^{1 / 2}$, where $\omega_{2}$ is the plasma frequency of the gas surrounding the radiator. For multiple interfaces, interference effects may lead to an increase of the number of maxima and even to a change of the position of the most probable angle, and remove the strong dependence on the $\gamma$-factor. Previous attempts to measure the TR angular distribution were not fully successful because of the limited functionalities of the setups [6-8]. In this work, for the first time high precision simultaneous measurements of the TR photon energy and its production angle have been performed. This was achieved using a silicon pixel detector readout by a Timepix3 chip [9].

\section{Experimental setup}

The experiment was carried out at the SPS H8 beam line at CERN. A few types of beams were used for these tests: $20 \mathrm{GeV}$ mixed electrons/pions, and muons with momenta of 120 and $180 \mathrm{GeV}$. A schematic view of the setup is shown in Fig. 1. In order to separate TR from the beam particle, the radiators were installed $2 \mathrm{~m}$ away from the Timepix3 based silicon detector. A pipe filled with helium between the radiator and the detector was used to minimize the absorption of the X-ray quanta along the path. Triggers corresponding to particles of different types were produced by a particle identification (PID) system consisting of an upstream Cherenkov counter (not shown in Fig. 1), a

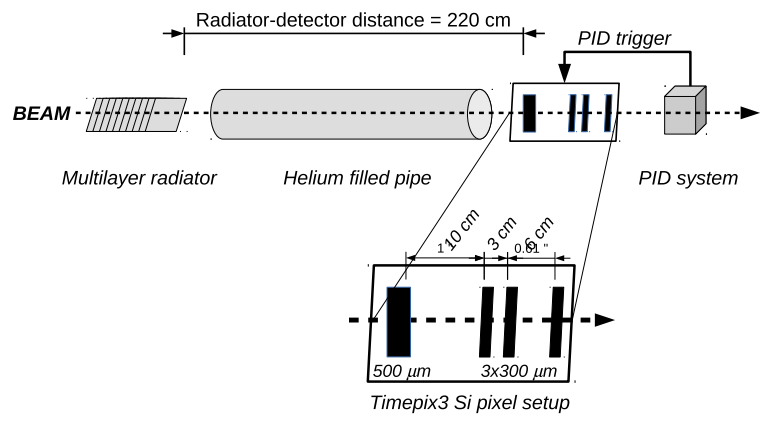

Fig. 1. The experimental setup.

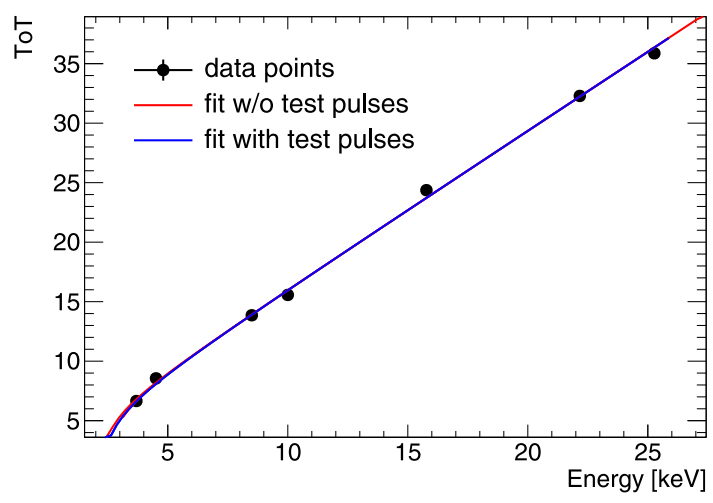

Fig. 2. Typical calibration fit for a single pixel.

preshower detector and a lead glass calorimeter. A multiplicity counter was used as veto to remove upstream showers and multiparticle events. These triggers were used to flag electrons and pions in the mixed beam operation. Different types of radiators, made of several materials and with different numbers of layers, were employed to broaden the space of possible configurations to be investigated. A systematic analysis of these results will be presented in a later publication.

The TR events were detected by a $500 \mu \mathrm{m}$ thick silicon sensor, with a pixel size of $55 \times 55 \mu \mathrm{m}^{2}$, bump bonded to a Timepix 3 chip. Such a sensor provides enough stopping power to absorb the expected TR spectrum. Timepix3 [9] is a pixelated readout chip produced in $130 \mathrm{~nm}$ CMOS technology for a broad range of applications. Hybridization with a semiconductor sensor makes it an optimal detector for high energy particles and X-rays. For this experiment, Time-over-Threshold (ToT) information for signals from each pixel provides an accurate measurement of the energy deposition in each cluster. The ToT scale of the Timepix3 detector was calibrated in the laboratory previous to the test beam. The calibration was performed using X-ray fluorescence in an energy range between $3.7 \mathrm{keV}$ (calcium $\mathrm{K}_{\alpha}$ line) and $25.4 \mathrm{keV}$ (silver $\mathrm{K}_{\beta}$ line), on a per-pixel basis. To adequately cover the low energy region, internal test pulses were employed. The calibration data points were fitted by a function of the form [10]

ToT $=a E+b-\frac{c}{E-E_{0}}$

where $E$ is the photon energy and $E_{0}$ is the photon energy at ToT $=0$. An example of a single pixel calibration fit is shown in Fig. 2. The measured cluster energy resolution follows the formula

$R(E)=0.9022 \times E^{-0.887}$

where $E$ is measured in $\mathrm{keV}$. The Timepix3-based silicon pixel detector was followed by a single arm of the LHCb VELO telescope [11], 


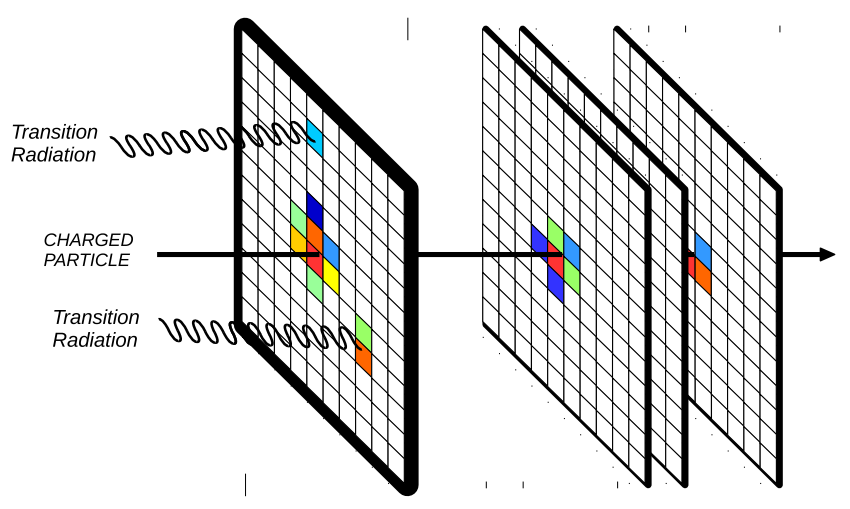

Fig. 3. Sketch of a transition radiation event in the Timepix 3 system.

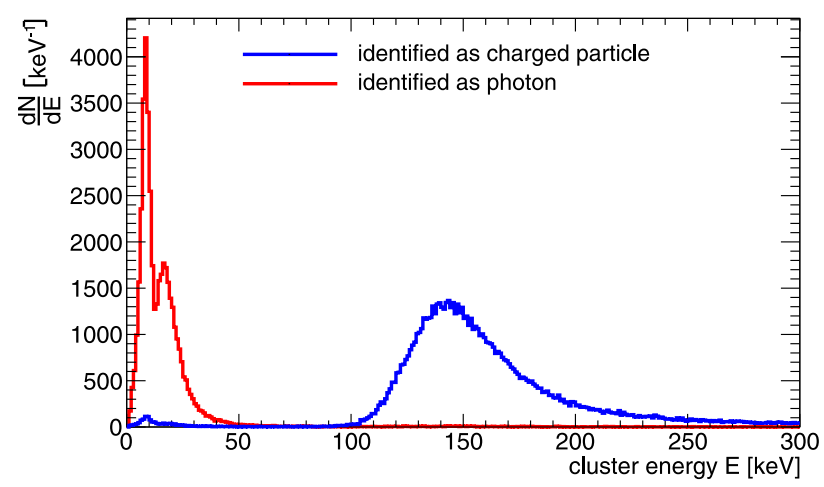

Fig. 4. Distributions of energy for all the clusters identified as the charged particle (blue) and as transition radiation (red) in events with an electron trigger. (For interpretation of the references to color in this figure legend, the reader is referred to the web version of this article.)

consisting of 3 planes of pixel sensors bump bonded to Timepix 3 chips. This provides tracking information that can be used to distinguish the particle cluster from the X-ray clusters in each detection event. The $1+3$ planes were integrated in the same SPIDR readout system [12,13], so that the data from the front sensor was automatically synchronized with the data from the tracking system. The electron-pion trigger coming from the PID system was fed to the SPIDR readout, which would add it as a flag to the output data.

\section{Analysis of the data}

Fig. 3 schematically represents an event with TR photons accompanying the particle. The X-rays eventually emitted in the radiator, travel at a small angle along the path of the original charged particle inside the Helium filled pipe. If the distance between the charged particle and a photon is large enough, they produce well separated clusters of pixels when absorbed by the first $500 \mu \mathrm{m}$ thick silicon sensor. While the X-rays are mainly absorbed by the front sensor, the charged particle continues its path and leaves a track in the following 3 pixel planes, which is reconstructed offline. Cluster reconstruction, noisy-pixel masking, alignment and tracking were performed using the Proteus software package [14]. The distribution of the residuals between the track prediction and the center of the particle cluster on the front sensors gives an RMS of $\sim 30 \mu \mathrm{m}$ in both directions on the sensor surface. A typical cluster size (number of pixels in cluster) is 5-6 for particles and 1-2 for TR photons. Photon clusters are considered to be identified if they are separated by a distance of one pixel from the particle cluster. Cluster energy is a very clear separation parameter. It turns out that in order to achieve separation between the photon and particle clusters, it is sufficient to identify the latter as the most energetic cluster in the event. This is shown in Fig. 4, where the two spectra appear clearly separated.
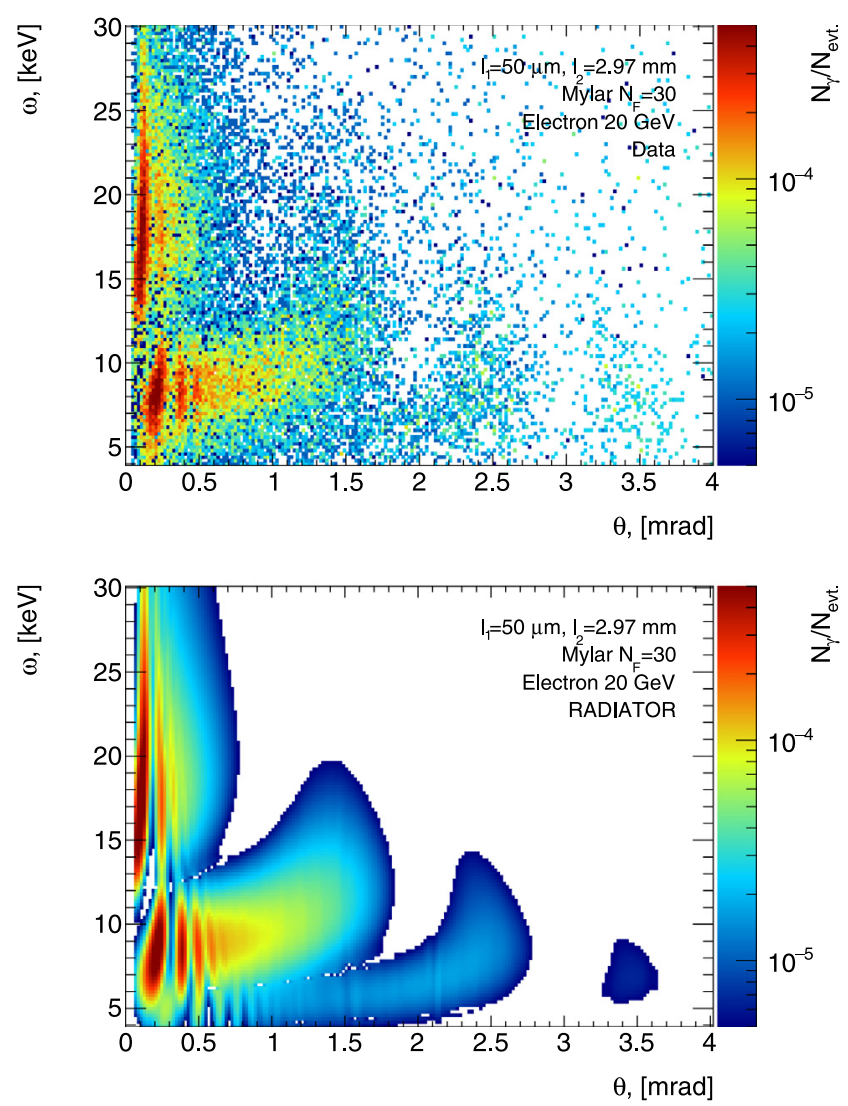

Fig. 5. Energy versus angle distribution of TR photons for Mylar radiator consisting of 30 foils of $50 \mu \mathrm{m}$ thick sparated by $2.97 \mathrm{~mm}$ : data (top) and simulation (bottom).

\section{First TR measurement results}

Once the photon and particle clusters are identified, their distance is calculated. At this point, each TR cluster is flagged with the values of its energy and emission angle. An example of a two dimensional distribution of the absorbed photons is shown in Fig. 5 for both data and simulation. The results shown in these figures were obtained with a radiator consisting of 30 layers of Mylar foils of $50 \mu \mathrm{m}$ thickness separated by $2.97 \mathrm{~mm}$. A simplified model of the detector was used in the simulations. TR was simulated according to the approach described in [15]. This allows one to calculate the energy spectra of TR. The absorption in the radiator materials was also taken into account. Once the spectra are defined, the angular distribution of individual photons was calculated using the formulas described in [5]. The energy-angle distribution shows the typical structure expected for TR. It includes global components, filling large areas, and fine components, creating additional interference patterns. The global component arises from the properties of a single foil (material and thickness) and it accounts for the interference of the TR photons produced at two foil interfaces. The fine structure is defined by interference effects between multiple foils and manifests itself as a modulation of the global structure. The TR photons can be separated in two parts: low energy $(<14 \mathrm{keV})$ and high energy $(>14 \mathrm{keV}$ ), which have different angular distributions. At high energy, the $\mathrm{X}$-rays are produced at small angles following the law $\theta=\left(1 / \gamma^{2}+\omega_{2}^{2} / \omega^{2}\right)^{1 / 2}$. At low photon energies the angular distribution is wider. These two components of the TR have different $\gamma$ dependences [4]. The generation of photons at high energy starts at higher gamma factors than for the lower energy photons. Fig. 6 shows the projections of the $2 \mathrm{D}$ distribution along the two axes. Data and simulations are shown on the same plots. A good agreement between simulation and data is found. Small differences at energies above 14 $\mathrm{keV}$ may be explained as an overestimation of the resolution between 

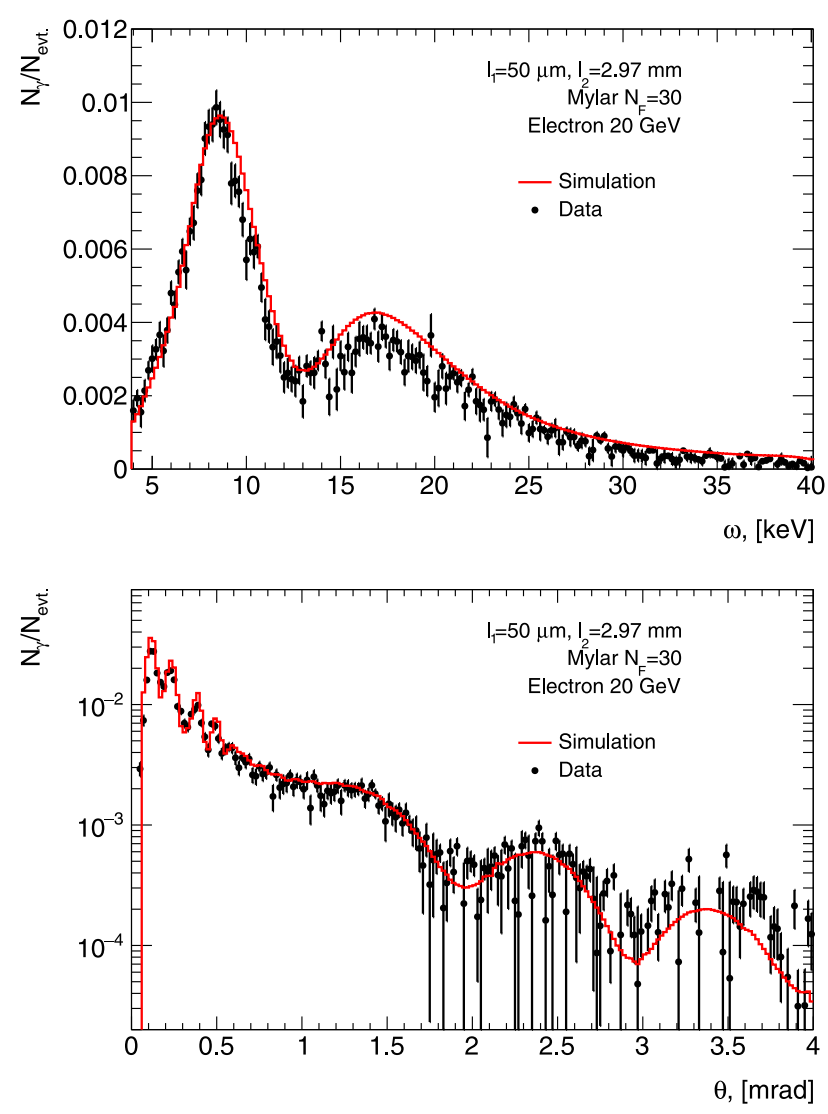

Fig. 6. Data-simulation comparison, energy projection (top) and angle projection (bottom).

close-by photon clusters and particle clusters in the simulation, and are the target of current studies.

\section{Conclusion}

Thanks to the excellent combined spatial and energy resolution of a silicon Timepix 3 detector, first detailed studies of the TR angle-energy spectra have been performed at the CERN SPS test beam facility. A preliminary analysis of the data produced the very first high resolution images of the energy-angle phase space of TR. The characteristic interference patterns foreseen by the theoretical models are made visible with an unprecedented level of detail. These results not only shed light on possible future techniques for exploiting TR for particle identification at high Lorentz factors, but are also extremely useful for understanding the theory behind TR.

\section{Acknowledgment}

We gratefully acknowledge the financial support from Russian Science Foundation grant (project No. 16-12-10277).

\section{References}

[1] B. Dolgoshein, Transition radiation detectors, Nucl. Instrum. Methods A 326 (1993) 434-469.

[2] A. Andronic, J.P. Wessels, A. Andronic, J.P. Wessels, Transition radiation detectors, Nucl. Instrum. Methods A 666 (2012) 130-174.

[3] C. Patrignani, et al., (Particle Data Group), Transition radiation detectors, Chin. J. Phys C40 (2016) 100001.

[4] N. Belyaev, et al., Test beam studies of possibilities to separate particles with gamma factors above $10^{3}$ with straw based Transition Radiation Detector, J. Phys. Conf. Ser. 934 (2017) 012053.

[5] M. Cherry, Measurements of the spectrum and energy dependence of X-ray transition radiation, Phys. Rev. D 17 (9) (1979) 2245.

[6] M. Deutschmann, et al., Particle identification using the angular distribution of transition radiation, Nucl. Instrum. Methods 180 (1981) 409.

[7] S. Furletov, J. Furletova, Geant4 simulation of transition radiation detector based on DEPFET silicon pixel matrices, Nucl. Instrum. Methods 706 (2013) 73-78.

[8] N. Belyaev, et al., Measurements of angular distribution and spectrum of transition radiation with a GridPix detector, J. Phys. Conf. Ser. 934 (2017) 012049.

[9] T. Poikela, et al., Timepix3: a 65K channel hybrid pixel readout chip with simultaneous ToA/ToT and sparse readout, JINST 9 (2014) C05013.

[10] J. Jakubek, Precise energy calibration of pixel detector working in time-overthreshold mode, Nucl. Instrum. Methods 633 (2011) S262-S266.

[11] D.M. Saunders, The Timepix3 Telescope for LHCb Upgrade RD 1 measurements, Proceedings, 38th International Conference on High Energy Physics (ICHEP 2016): Chicago, IL, USA, August 3-10, 2016.

[12] B. van der Heijden, et al., SPIDR, a general-purpose readout system for pixel ASICs, JINST 12 (2017) C02040.

[13] J. Visser, et al., SPIDR: a read-out system for Medipix3 \& Timepix3, JINST 10 (2015) C12028.

[14] https://gitlab.cern.ch/unige-fei4tel/proteus/.

[15] P. Nevski, Advances in the simulation of Transition Radiation Detectors, Nucl. Instrum. Methods A 522 (2004) 116. 